\title{
Impfen - ein emotional besetztes Thema
}

Zurzeit versuche ich wieder - und bisher immer noch vergeblich - eine meiner Nichten davon zu überzeugen, wenigstens jetzt nach der Geburt ihres 2. Kindes die Gelegenheit zu nutzen und beide Kinder gegen Masern impfen zu lassen. „Deutschland wegen Masernepidemie gerügt" war deshalb eine der Schlagzeilen der letzten Wochen, die mich ganz persönlich angesprochen hat. Die WHO hat zwar ein paar Jahre gebraucht, bis sie die Masernepidemie in Duisburg 2006 zu einem Thema machte, aber betroffen macht das trotzdem.

Surfen Sie einmal zu diesem Thema im Internet: Auch die unter Ihnen, die nicht täglich Mütter zu überzeugen versuchen, ihre Kinder impfen zu lassen, werden sehr schnell merken, dass dieses Thema ähnlich dogmatisch und emotional diskutiert wird wie beispielsweise das Thema Fluglärm. Ich denke, wir sollten dem Thema Impfen und medikamentöse Prophylaxe - natürlich mit dem Schwerpunkt Reisen - in nächster Zeit ein Sonderheft widmen und uns dabei gezielt auch mit Impfmüdigkeit und alternativmedizinischen Inhalten und Verfahren befassen. Einen Anfang machen wir bereits in dieser Ausgabe mit einem Beitrag von Dr. Dr. Peter C. Döller zu den Impfempfehlungen für eine Weltreise. Fakten und Argumente zusammenfassend darstellen und somit zu kennen wird zwar das Problem nicht lösen, aber hoffentlich zur Versachlichung der Diskussion beitragen und auch Argumentationshilfen liefern. Wie immer sind Sie selbst aufgerufen, Fallbeispiele und Artikel für unsere Zeitschrift zu verfassen. Machen Sie das doch einmal zum Thema Impfmüdigkeit!

Für mich wird dieses Editorial wohl mein „Abschieds-Editorial“, weil mein Turnus als Vorsitzender des Fachverbands Reisemedizin und somit auch die Mitgliedschaft im Editorial Board dieser Zeitschrift zu Ende geht. In gewisser Weise hat ja die Flugmedizin Tropenmedizin
Reisemedizin das Erbe des Kongresses „Medizin und Mobilität" angetreten und versucht, alle Gesellschaften zu vertreten, die sich mit der Thematik „Medizin in der mobilen Gesellschaft" befassen. Natürlich bleibe ich der Thematik und auch der Zeitschrift treu und werde sie weiterhin unterstützen.

In diesem Jahr werden sich einige Terminkollisionen nicht vermeiden lassen: Am 18. und 19. September findet sowohl die Jahrestagung des Deutschen Fachverbandes Reisemedizin in Göttingen als auch die Jahrestagung der Deutschen Gesellschaft für Luft- und Raumfahrtmedizin in Fürstenfeldbruck statt. Auf das gleiche Wochenende hat der Vorstand des Deutschen Zentrums für Luft- und Raumfahrt auch den „Tag der Luft- und Raumfahrt“ in Köln gelegt. Für mich etwas schwierig zu organisieren, ich sollte mich „dreiteilen“, doch für viele von Ihnen wird das bedeuten, dass eine der 3 Veranstaltungen nicht allzu weit von Ihnen entfernt stattfindet. Also: Nutzen Sie die Gelegenheit und kommen Sie an diesem Wochenende zu einer der 3 Veranstaltungen, denn Kennenlernen und fachliche und persönliche Kontakte zu vertiefen ist wichtig. Die Jahrestagungen der übrigen Gesellschaften können Sie ja hoffentlich ohne Terminkollisionen besuchen.

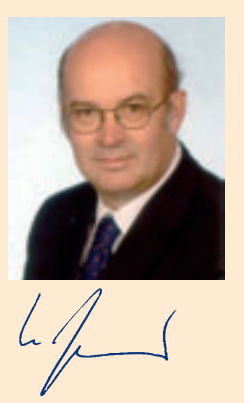

Prof. Dr. Rupert Gerzer, Köln 